\title{
Probabilistic Edge Weights fine-tune Boolean network dynamics
}

Dávid Deritei ${ }^{1,2, *}$, Nina Kunšič ${ }^{1}$, Péter Csermely ${ }^{1}$

1 - Department of Molecular Biology, Institute of Biochemistry and Molecular Biology, Semmelweis University, Budapest, Hungary

2 - Channing Division of Network Medicine, Brigham and Women's Hospital, Harvard Medical School, Boston, USA

* - corresponding author. Contact at: david.deritei@channing.harvard.edu

\section{Abstract}

Biological systems are noisy by nature. This aspect is reflected in our experimental measurements and should be reflected in the models we build to better understand these systems. Noise can be especially consequential when trying to interpret specific regulatory interactions, i.e. regulatory network edges. In this paper, we propose a method to explicitly encode edge-noise in Boolean dynamical systems by probabilistic edge-weight (PEW) operators. PEW operators have two important features: first, they introduce a form of edge-weight into Boolean models through the noise, second, the noise is dependent on the dynamical state of the system, which enables more biologically meaningful modeling choices. Moreover, we offer a simple-to-use implementation in the already well-established BooleanNet framework. In two application cases, we show how the introduction of just a few PEW operators in Boolean models can fine-tune the emergent dynamics and increase the accuracy of qualitative predictions. This includes fine-tuning interactions which cause non-biological behaviors when switching between asynchronous and synchronous update schemes in dynamical simulations. Moreover, PEW operators also open the way to encode more exotic cellular dynamics, such as cellular learning, and to implementing edge-weights for regulatory networks inferred from omics data.

\section{Introduction}

Boolean network models or Boolean dynamical systems have become a standard toolkit for modeling biological systems of increasing size and complexity [1-7]. The main advantage of using Boolean models is that they offer a reasonable compromise in complexity: the regulatory mechanisms and interactions are expressed through logical rules, while the number of parameters remains manageable, even in large systems. The dynamical side of Boolean models is key: the attractors of validated models correspond to stable phenotypes of biological systems $[8,9]$. Such a representation of phenotypes can help to understand the underlying mechanisms of the behavior such as biological phenotypes emerging from local interactions, mutations leading to pathological phenotypes, etc. $[10,11]$. Boolean models can also help in 
identifying the key regulatory circuits associated with phenotypic decision-making [12,13] and even help identify control targets in order to drive the system into phenotypes (e.g. to switch from an unhealthy state to a healthy one) [14-16]. Boolean models address questions that lie at the core of modern systems biology, but also of modern medicine and drug development [2].

A large family of Boolean Network models is concerned with modeling biological noise and uncertainty coming from incomplete measurements or sparsity of data. Some of these methods introduce uniform noise on a system level (e.g. perturbed Boolean Networks), introduce noisy function selection on the level of nodes (e.g. Probabilistic Boolean Networks [17], Dynamic Bayesian Networks [18], Stochastic Discrete Dynamical Systems [19]), or combine system- and node-level noise (perturbed Probabilistic Boolean Networks) [20]. In this work, we introduce a method that applies noise on the level of individual edges or hyper-edges (edges defined between sets of vertices) in a biologically meaningful way, through probabilistic edge weight (PEW) operators. Generally, PEW operators are mathematical objects that can be added to the regulatory rules of Boolean models to modulate the noisiness of edges. On one hand, PEW operators offer a way to handle edge-level uncertainty, on the other, they offer a means to introduce relative edge-strength within a system. Moreover, the noise level can be made a function of the system's dynamical state, which is not a common way of introducing noise in Boolean systems, despite the evidence in literature that in fact noise can be highly dependent on the dynamical state and environment of the cell [21-23]. For example the dynamical behavior of a node can fluctuate when its regulators are present but their molecular interactions are noisy. In contrast, it's less likely to fluctuate from an off state when its regulators are absent. In the next sections, we place the PEW operator framework within the context of other noisy Boolean models and demonstrate through two empirical examples that introducing PEW operators to the Boolean model improves its predictive capabilities.

\section{Boolean dynamical systems - definitions}

Boolean regulatory networks can be represented by a graph $G=(V, E)$ consisting of $V=\left(v_{1}, v_{2}, \ldots, v_{N}\right)$ vertices and $E=\left(e_{i j} \mid i, j \in V\right)$ directed edges. Each vertex (node) has a binary state, $\sigma_{v}$ equal to 1 or 0 , often referred to as ON or OFF. The state of the system is the collective state configuration of all of its constituent nodes in time $t$. Overall, the model can have $2^{N}$ different states, where $N$ is the number of vertices (nodes). The state of each node $v$ is determined by a unique logical function assigned to it, $F=\left(f_{1}, f_{2}, \ldots, f_{N}\right)$. We also call these functions Boolean regulatory functions or Boolean rules. The logical function encodes how every node responds to the different state-combinations of its regulators. The inputs of each function $f_{i}$ are the states of the regulators (nodes with edges pointing to) of $v_{i}$ in time point $t$. The value of a node $\sigma_{v_{i}}$ in time-step $t+1$ is calculated as:

$$
\sigma_{v_{i}}(t+1)=f_{i}\left(\boldsymbol{\sigma}_{\operatorname{Par}\left(v_{i}\right)}(t)\right)
$$


where $\operatorname{Par}\left(v_{i}\right)$ is the set of parents (regulators) of node $v_{i}$ and $\boldsymbol{\sigma}_{\operatorname{Par}\left(v_{i}\right)}(t)$ is their state configuration at time-point $t$.

Different update schemes determine the order in which the functions are evaluated, i.e. the state of the nodes is updated. In the case of the synchronous update scheme, all nodes are updated at the same time, such that the state of the system in time $t$ fully determines the state of the system in time $t+1$. This results in deterministic trajectories, where the emergent dynamics of the system depend only on the initial configuration. Asynchronous update schemes update the system one node at a time and emulate different (more granular) time scales as compared to synchronous update schemes. This is mainly because in the case of synchronous update one time-step represents $N$ node updates, while in the case of asynchronous updates, one time-step represents only one node update. There are exceptions when $t$ is incremented only after all nodes were updated at least once asynchronously. A certain degree of stochasticity can be added to the system dynamics with randomized update schemes. Random order asynchronous update picks nodes from a shuffled list of the nodes, updating each once before reshuffling, while general asynchronous update picks nodes in a truly random way, allowing repetition.

The attractors of a Boolean system represent the long-term equilibrium states of their dynamics. Fixed-point attractors or steady states are states in which all logical functions are satisfied and updating nodes no longer changes the state of the system. Attractors can also be limit cycles or complex attractors, which, instead of a single state, are a set of states that the system keeps visiting indefinitely (a.k.a. an ergodic subset of states).

\section{Boolean models with stochastic properties introduce different varieties of noise}

Scientists have realized early that completely deterministic Boolean networks (BNs) are limited in their capability to model real systems $[2,17,19,20,24]$. There is indeed a spectrum of noise sources in a biological system, from basic thermodynamic noise to heritable genetic differences (mutations) among individual cells of a modeled cell population; there is also measurement noise as well as uncertainty due to lack of data $[25,26]$. Mirroring this variety in sources of noise of real systems, there are many types of noise one can introduce into a BN.

One of the earliest types of noisy BN were the perturbed Boolean Networks (BNp) [27]. The noise in BNps is added by a perturbation of a random node-flip with a nonzero probability $p$ in each time step. Essentially, before each node update one tosses a coin with bias $p$, which determines whether the next value of the node is going to be determined by its deterministic function or it's going to flip to its other state, regardless of its function. This simple modification turns BNps into ergodic Markov Chains, which adds a number of advantages to their use and analysis. For instance, attractors in BNps still carry most of the probability mass but the system can leave the attractors with a nonzero probability. One of the drawbacks of BNps is that they 
introduce a very general "thermodynamic" stochasticity that does not give much freedom in fine-tuning node-specific aspects of the noise.

One of the most prominent families of noisy BNs is Probabilistic Boolean Networks (PBNs) introduced by Shmulevich et al. [17], which address the lack of node-specificity of BNps. PBNs have a set of functions assigned to each node (instead of a single unique logical function). The different possible combinations of selected functions give rise to different "realizations" of BNs. The total number of realizations is equal to the product of function set sizes. At each time update, a random binary variable $\xi$ decides whether a new realization shall be used, or the BN remains the same. If $\xi(t)=1$ new functions are chosen for each node from their individual pool of functions. The functions from each set are picked with a pre-determined probability (adding up to 1 within each set).

PBNs do not necessarily constitute ergodic systems, however, they can contain ergodic subsets of states akin to complex attractors. One can also combine perturbation noise with PBNs (PBNp), this way there is a non-zero probability of nodes committing "mistakes" and also changing their function. PBNs have a wide range of applications and are a popular model of biological systems [27]. Although they are very versatile, an inherent limitation of PBNs is that the noise parameters are independent of the state of the system. In contrast, we often see in biological regulation that the state of the system greatly influences the degree of noise $[21,22]$.

Another popular noisy model that incorporates a dependence of the noise on the system state is the stochastic discrete dynamical system (SDDS) introduced by Murrugarra et al. [19]. Instead of a set of functions (like in PBNs), nodes in SDDSs are assigned a single function and two probability values: an activation and a degradation propensity ( $p_{u p}$ and $p_{\text {down }}$ ). The two propensity values determine whether a node "accepts" its new update value or remains the same. If a node's current value $x(t)$ is smaller than its update value, $f(x(t))$, it means $x$ is up-regulated, thus the up propensity $\left(p_{u p}\right)$ will determine by a biased coin-toss if the $x(t+1)$ will be equal to $f(x(t))$ or to $x(t)$. The case of down-regulation works the same way in the case of $x(t)<f(x(t))$, with $p_{\text {down }}$ determining the bias of the coin-toss.

A significant innovation of SDDSs, as alluded to earlier, is that the noise is dependent on the value of the node at time $t$, and also the probabilities are not necessarily symmetric (e.g. $p_{\text {up }}^{i}+p_{\text {down }}^{i} \neq 1$ ). This might not carry the same mathematical elegance as previous models but is very useful biologically, where such asymmetries are common. The method presented in this paper has a similar principle as the one introduced by Murrugarra et al. [19] in that the noise is dependent on the state of the system and is not symmetrical.

Murphy et al. have shown [18] that Boolean networks are indeed a special case of a broader class of Dynamic Bayesian Networks (DBNs). DBNs are defined by a set of nodes that represent random (hidden or known) variables, and directed links are described by the conditional dependence between the variables. The value of each node is determined by a conditional probability distribution (CPD), dependent on the parents of the node. Dynamic 
Bayesian networks are a special case of the general Bayesian networks, where the dynamic aspect is encoded by a different set of $N$ nodes representing each timestep. If all nodes have deterministic logical functions with a binary output then the DBN is a Boolean network.

We would argue that the PEW method is a step toward the DBN framework in generality because the rules of some nodes with PEWs become stochastic and conditionally dependent on the value of the (subset of) parents. Nonetheless, it's still more pragmatic to view PEW models as a separate framework because, as we show in the empirical applications, probabilistic edge weights are meant as very specific, targeted modifications to Boolean models and are easily made compatible with existing tools of Boolean network analysis. On the other hand, DBNs are likely the most general modeling framework of which PEWs models represent a special case.

Finally, the work of Poret et al. [28] is worth mentioning, even though their paper has not been published in a peer-reviewed journal. Their method is close in spirit to what is proposed in this paper, and they provide great examples of fine-tuning BN dynamics with different kinds of noise. In their work Poret et al. introduce "fuzzy operators" in their logical functions, which are capable of evaluating continuous node-states. The use of "fuzzy operators" allows the method to fine-tune edge responsiveness and edge reactivity, all of which are indeed quite nuanced interactions.

We have used a similar weakening/strengthening approach on the level of nodes in one of our recent papers [29] and its follow-up study [30], where we imposed external perturbations to specific nodes, especially input nodes, setting them to a certain Boolean value with probability $p$ in every time-step. This helps to establish a continuous "concentration"-like parameter, which can influence the downstream dynamics to a great degree. We use this same approach in this paper to alter the input concentration of TGFB in the applications to the EMT model.

In the following sections, we present the PEW method and we show a few empirical examples in which we demonstrate its usefulness in recapitulating and explaining experimental results. Finally, we demonstrate its versatility through reproducing examples from other stochastic methods. Indeed, BNp-s, SDDS (Boolean case), and the simulation results of Poret et al. [28] are all reproducible in the PEW framework (See Appendix 1 and the Supplementary Jupyter Notebooks).

\section{The PEWs offer an easy way to insert noise to individual edges in a Boolean network}

Let us denote the probabilistic weight operator as $P_{e}$, which has two parameters: $w_{u p}$ and $w_{\text {down }}$. We also associate a function $f$ to $P_{e}$, which describes how the weights determine the outcome of the operation. 
Generally:

$P_{e}\left(w_{o n}, w_{\text {off }}, f\right) x=\left\{\begin{array}{l}f\left(x, w_{\text {on }}\right), \text { if } x \geq \theta \\ f\left(x, w_{\text {off }}\right), \text { if } x<\theta\end{array}\right.$

Here $x$ represents the source of a (hyper)edge in the Boolean model. A hyperedge is an edge of a hypergraph, a generalized graph, where edges connect sets of vertices (undirected) or ordered subset pairs (directed) [31]. In a Boolean model, a hyper-edge is a clause of the Boolean function consisting of multiple nodes regulating the target node (e.g. A AND B). The $P_{e}$ operator used in a Boolean rule affects the first variable on its right-hand side. This variable can refer to a single node or to a clause of the Boolean rule involving multiple input nodes (in which case the operator is followed by parentheses, containing a subset of the logic function). Such clauses represent hyperedges, as they characterize the relationship between the target node and several of its inputs. The $\theta$ threshold parameter is 0.5 for the Boolean case, but for continuous or multi-level cases it can be adjusted.

The "grammar" of the $P_{e}$ operator is the same as of any left-hand side operator (such as "not") i.e. it acts on the first mathematical clause to its right. Below we illustrate the rationale and the exact details of how the PEW operator works in a simple example.

Consider the Boolean rule $A *=B$ AND $C$. In biological models, a function such as this usually encodes that gene $A$ is activated by a complex formed by $B$ and $C$. Both $B$ and $C$ are necessary for A's activation; thus $A$ will not be activated if $C=0$. Next, we assume that the link between $A$ and $C$ is weakened (e.g. due to a mutation), in other words, we decrease the weight of the $C \rightarrow$ A link. Adding a PEW operator we can represent this as $\mathrm{A}^{*}=\mathrm{B}$ AND $P_{e} \mathrm{C}$. The weight is decreased in a probabilistic way by making it noisier. The most extreme case of weakening an edge is cutting it entirely. In general, cutting a link in a Boolean network implies a choice between setting the state of the source of the link to 0 or 1 . These two choices have drastically different interpretations. Cutting the $C \rightarrow A$ link by setting $C=1$ yields the Boolean rule $A^{*}=B$, which indicates that the necessity of $C$ was eliminated altogether. If the $C \rightarrow A$ link is cut by setting $C=0$ then the Boolean rule becomes $A^{*}=0$, thus $A$ will be locked OFF. The mechanistic interpretation of $A^{*}=B$ AND $C$ helps explain the need to apply different levels of noise to the ON vs. OFF states of $C$. For instance, if $C$ is mutated so that it only binds $B 30 \%$ of the time, this probability should only be applied to the cases in which $C$ is $O N$, i.e. there is something to bind to $B$. If $C$ is OFF there is nothing even to attempt the complex formation, therefore $A$ will be OFF $100 \%$ of the time. This is the reasoning behind the conditional update in equation (1). In this example $w_{o n}=0.3$, and $w_{o f f}=0$.

As alluded to in the example above $f$ can be a simple draw from a binary distribution (i.e. a biased coin-toss) with probability $w$,

$f(x, w)=\operatorname{Pr}\{x=1, w\}$. In summary, $\mathbf{A}^{*}=\mathrm{B}$ and $P_{e}\left(f, w_{o n}, w_{o f f}\right) \quad \mathrm{C}$. This means that whenever $C$ is ON the noise weakens its effect to as though it were ON only $30 \%$ of the time and whenever it is OFF it is not affected by noise. 
Last but not least, the PEW operator is not restricted to acting on single edges, but it can act on any hyperedge. This means practically, that any clause of the Boolean rule can be made noisy with the PEW operator, and any number of PEW operators can be used. In our previous example, it is possible that the $\mathrm{B}+\mathrm{C}$ complex itself is noisy. In that case, $\mathrm{A}^{*}=P_{e}(\mathrm{~B}$ and $\mathrm{C})$ would make the joint effect of $B$ and $C$ noisy instead of a single one.

The application of targeted PEW operators on an ensemble of simulated systems leads to a fine-tuning of the concentrations of molecules, which otherwise behave non-biologically in the model. In the next sections, we will show the biological relevance of such fine-tuning.

\section{Implementation in BooleanNet}

One of our goals with this method is to offer an easy way to use PEW operators without the need for additional software. Thus the technical implementation is done as an extension of the already popular and well-established BooleanNet framework in Python [32]. In the modified branch of BooleanNet PEW operators are implemented within the rule-parsing grammar of the BooleanNet method (which uses the Yacc framework [33]) and work as left-hand side operators (the same as not). The syntax of a PEW operator in a Boolean rule is two positive floating-point numbers [w_on, w_off], separated by a comma within a square bracket. The noise function is specified separately (by default it is a Bernoulli coin toss). Following the example used previously:

$A^{*}=B$ and $C$ after applying the PEW operator becomes:

$A^{*}=B$ and $[0.3,0] C$

Or using the hyperedge:

$A^{*}=[0.3,0](B$ and $C)$

To use a PEW operator in BooleanNet only the Boolean rules of the simulated model have to be modified, following the syntax specified above. All other functions and operations (unless defined otherwise) follow the BooleanNet standards. The PEW operators also interact intuitively with the not operator, so using a PEW operator before or after a not has different effects. For example:

$A^{*}=[0.3,0]$ not $C$

has the same probabilistic outcome as

$A^{*}=\operatorname{not}[0.7,1] C$.

Generally, the not operator to the left modifies a PEW operator [w_on, w_off] to [1-w_on, 1-w_off]. A PEW operator to the left of a not, on the other hand, will act on the outcome of the negation. 
In this paper we follow the logic of the first number being associated with the ON case of the clause acted upon $(x)$ and the second number is associated with the OFF case. This, however, can be easily changed in the implementation, along with the threshold parameter $\theta$. Moreover, future versions of the framework can be expanded to the multilevel case, where instead of two values one can have a vector of arbitrary length, corresponding to all possible values of the input nodes.

\section{Applications}

In this section, we are going to demonstrate some applications of the PEW model, where the $\mathrm{PEW}$ enhanced $\mathrm{BN}$ performs better in explaining experimental results than the classic Boolean model, yet no complex ODE model is needed. To do this we switch from the more general $P_{e}\left(f, w_{o n}, w_{o f f}\right)$ notation to the more pragmatic [p_on, $p_{-}$off] notation, where the noise function is the Bernoulli coin-toss and the weights are determined as probabilities.

\section{Noisy feedback-loops explain the loss of $\mathrm{M}$ attractor stability in epithelial to mesenchymal transition (EMT)}

The transitions between epithelial and mesenchymal cellular phenotypes are encountered in embryonic development, wound healing, and cancer metastasis [34-36]. One of several proteins that trigger the signaling network that leads to epithelial-mesenchymal transition (EMT) is Transforming growth factor-beta (TGFB), an extracellular signaling molecule, which can trigger EMT but is also secreted by cells that underwent EMT. At the core of the EMT is a mutually repressive positive feedback loop between the Zeb transcription factors expressed in mesenchymal cells (M state), and the miR-200 microRNA expressed in epithelial cells (E state). This system acts as a bistable switch; once flipped from $E$ to $M$, cells lose expression of E-cadherin, a critical adherens junction molecule required for forming epithelial monolayers and maintaining an epithelial phenotype [6,16,37]. Celia-Terrassa et al. [38] showed in a fascinating study that a single mutation that weakened Zeb's ability to repress miR-200 expression radically altered the commitment dynamics of TGFB-induced EMT, and sped up the mesenchymal to epithelial transition (MET). Here we propose a very simple Boolean model, which qualitatively reproduces most of the results of the Celia-Terrassa study. Moreover, we show that the addition of two PEW-operators to the model significantly improves the model's qualitative results.

First, Celia-Terrassa et al. [38] showed that with increased TGFB concentration their cell lines exhibited a bistable behavior where the epithelial marker E-cadherin showed two distinct concentration peaks; one at a high concentration associated with a population of epithelial cells and one at a low concentration of a population of mesenchymal cells. This can be explained by the lock-in of the Zeb - miR200 mutual inhibition loop, which becomes self-sustaining 
(hysteresis). In Boolean modeling, we identify self-sustaining positive feedback loops as stable motifs [12], patterns of node activation that permanently lock in within the dynamics of the system. The authors created a separate cell line, where, using CRISPR technology, they weakened the Zeb-miR200 feedback loop by mutating the binding site of Zeb1 on miR200. This led to the disappearance of the previously observed bistability, where the E-cadherin peak changed linearly with increased TGFB concentration (instead of switching from low to high and never stabilizing between the two bistable peaks). In the following text, this Zeb1-miR200 edge knock-out line variant will be simply referred to as "mutant".

Second, they showed that in wild-type cell lines even a short (5 min), high concentration TGFB pulse can commit cells to EMT (even after washing off TGFB). The commitment only happened in mutant cells after a much longer $(1 \mathrm{~h})$ pulse, suggesting some other self-sustaining feedback loop downstream (other than the ZEB-miR200), which gets activated only with a longer pulse. Third, the authors showed that mutant cells left unperturbed transition back into the epithelial state much quicker ( $\sim-6$ days) than wild-type cells ( 12 days). The authors proposed a simple 4 node ODE model which explains the bistable behavior due to the ZEB-miR200 feedback loop and other related phenomena [38]. In this paper, we show that most results can also be explained using a simple PEW-enhanced Boolean model, with a minimal number of parameters.

There are a number of well established Boolean models for EMT [6], however, for the reproduction of these results we propose a simplified model with an important update to previous models, namely, that TGFB has to have a shorter, direct path to ZEB, which does not involve SNAI1 (in Steinway et. al. [6] all TGFB - ZEB paths go through SNAI1). The evidence that supports this shortcut is clear in the order of activation in response to the TGFB signal reported in the Celia-Terrassa paper (Fig 3C in [38]): SNAI1 turns on 5 hours after ZEB1. In our model, we represent this delayed signal from TGFB to SNAl1 with $n$ artificial intermediary nodes. We model the delayed feedback loop suggested by the experimental evidence with a SNAI1 local positive feedback loop; such feedback loop has support in the literature (see Appendix 2). We also add an alternate inhibitory pathway from TGFB to miR200 with $m<n$ intermediary nodes. For the Boolean rules of the model and additional references see Appendix 2. 


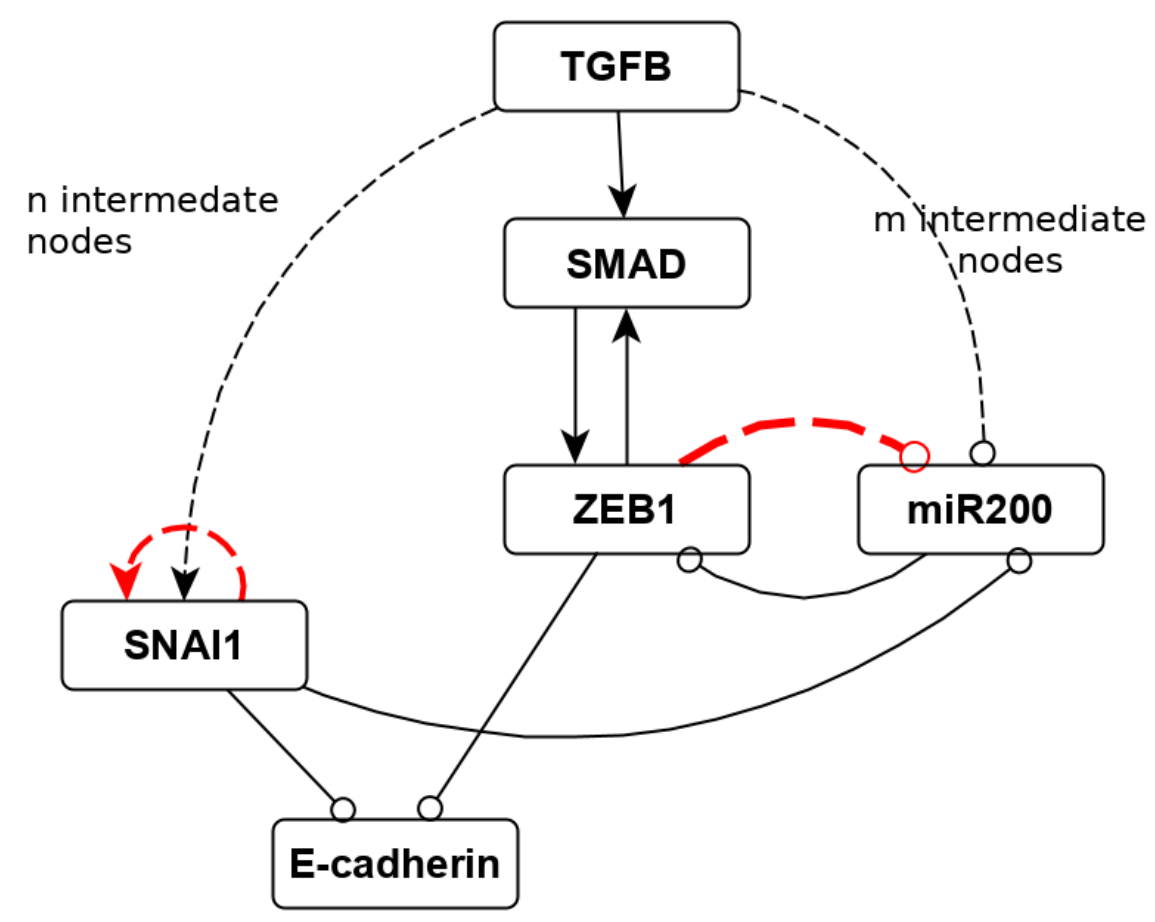

Figure 1: A simplified Boolean model of the EMT with PEW edges. Compared to another popular Boolean model of the EMT [6] this version has a shortcut from TGFB to ZEB (independent of SNA11) which we implemented through SMAD (evidence for a direct path in [39]). This shortcut is also justified by SNAl1's delayed activation compared to ZEB after the initial TGFB pulse in [38]. Edges ending in circles represent inhibition, edges ending in arrows represent activation. The black edges with dashed lines represent chains of intermediate dummy nodes which emulate the delayed signal $(n=6, m=3)$. The red dashed edges represent the feedback edges that have noise operators applied to them in both the wild type and the mutant case.

One potential disadvantage of Boolean models is that self-sustaining feedback loops (stable motifs) lock in permanently in the model dynamics, not encapsulating the fact that often there is a natural decay in the self-sustaining nature of the feedback loop after the termination of the initial signal. Simulating the model proposed above with classical Boolean dynamics permanently locks it into the mesenchymal state, given a long enough initial TGFB pulse. With the PEW framework, we can make stable motifs slightly more reversible, resulting in more nuanced dynamics. In the "wild type" version of our model, we add a very slight noise to the edges highlighted in red in Figure 1, namely to the local feedback loop of SNAI1 and the ZEB miR200 link. The consequence of this noise is that after the termination of the TGFB signal the system will slowly converge back into the epithelial state (i.e, it will undergo MET). Simulations with an ensemble of Boolean networks are shown in Figure 2. In Figure 3 we show that despite the edge noise the bistability due to the positive feedback loop is still maintained, i.e. the noise does not destroy the nonlinear effect of the transition. 
In the "mutant" version of our model instead of severing the ZEB - miR200 edge completely we create an almost severed noisy edge, obilassuming that the mutation does not fully abolish Zeb's ability to repress miR200. This is also a great example of why the noise should be dependent on the state of the node: When ZEB1 is ON there is still a slight chance for it to inhibit miR200 (imperfect loss of repression due to the mutation), but when it is OFF (e.g., in the absence of a TGFB signal), the chance of it turning miR200 OFF should be 0 (as is in the wild-type case).

Figures 2 and 3 show two additional model results that recapitulate the experimental data: first, the MET is significantly quicker in the mutant system compared to the wild type, and second, the mutant version loses its bistability, shown by the red median line of the E-cadherin distribution averaged in the interval of timesteps between 100 and 150.
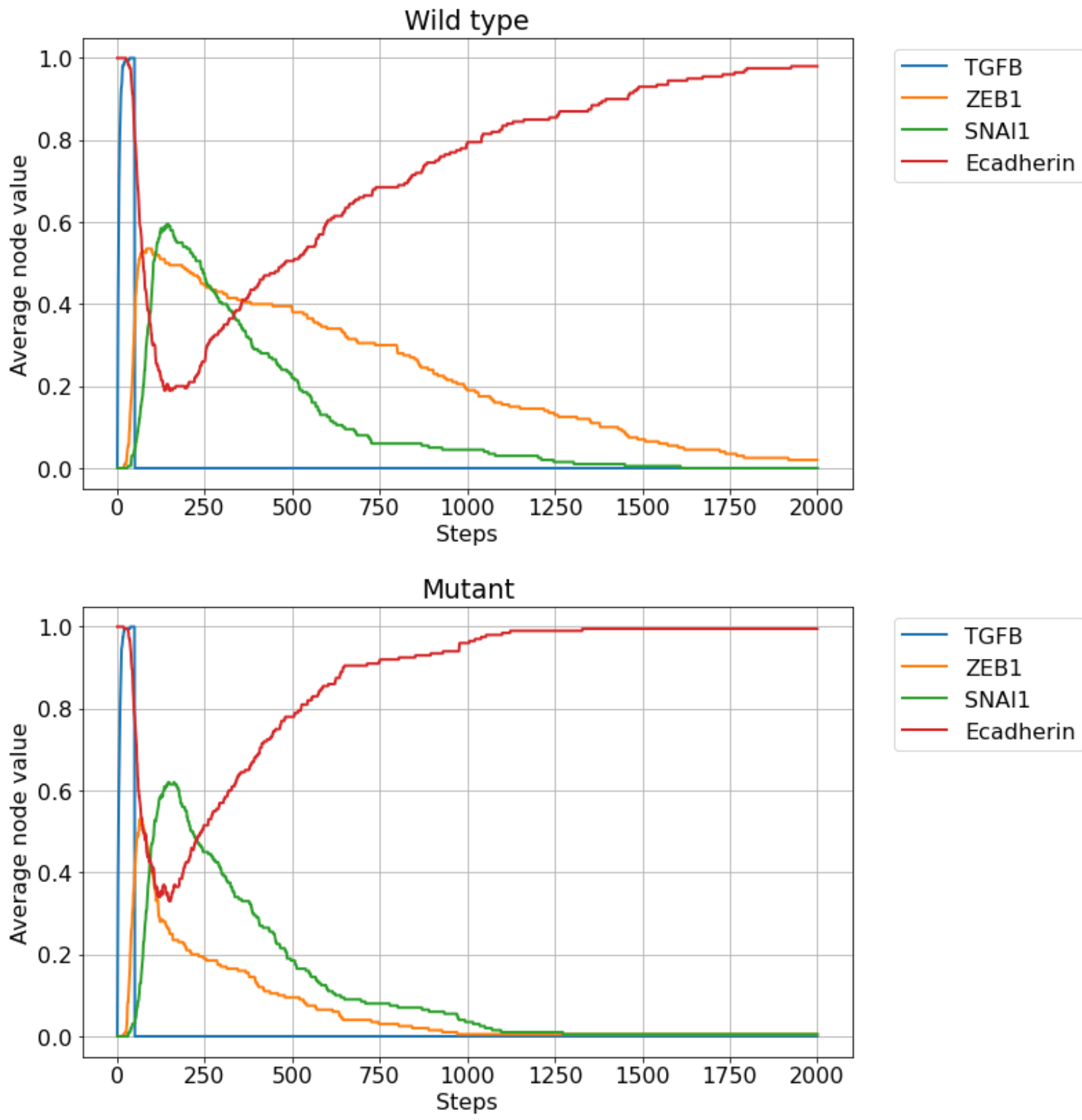
Figure 2: The "mutant" model has a faster MET compared to the wild-type version. Both panels represent the average node values of 100 independently simulated EMT models with a single strong initial TGFB pulse. The wild-type model (top) has a slight noise on both edges highlighted in red in Figure 1 (p_on=0.95, $p_{-}$off $=0$ ). The mutant (bottom) version has a stronger noise on the Zeb -- miR200 edge ( $p \_o n=0.05$, p_off $=0$ ) making the link almost severed. Due to the noise on the feedback loops, both assemblies return to the epithelial attractor, but the mutant does it a few hundred steps sooner due to the faster loss of ZEB, qualitatively matching the experimental results of [38].

Wild type
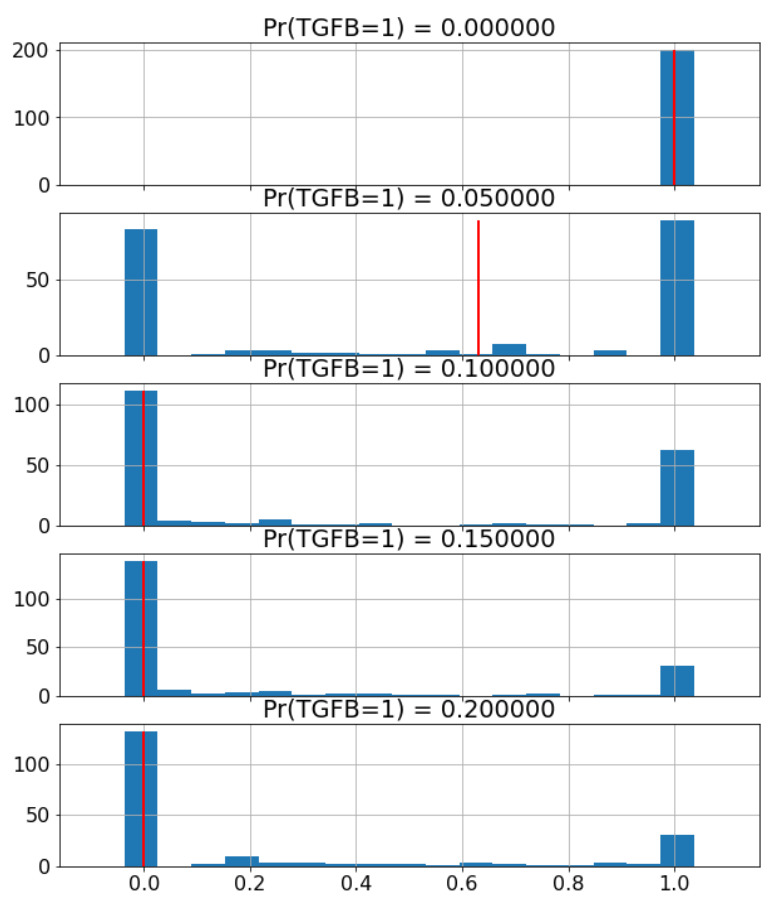

Mutant
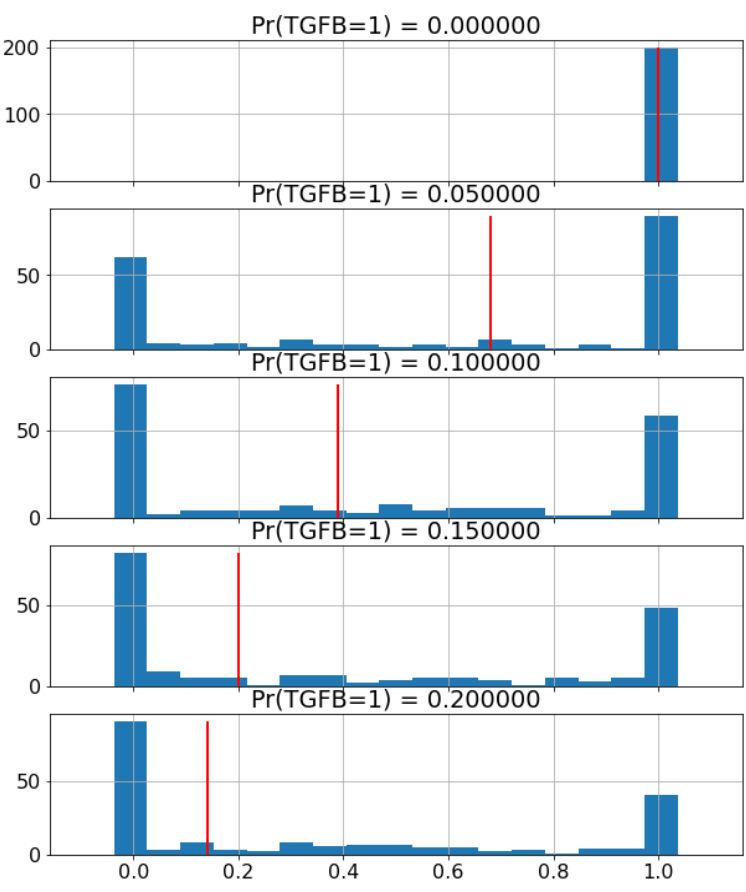

Figure 3: The mutant EMT model loses its nonlinear bistability in the function of the TGFB pulse concentration. The figures show the distribution of average E-cadherin between steps 100-150 in the models of wild type (left) and mutant cells (right). The red vertical line represents the median of the distribution. Despite the noisy edge the wild type still exhibits a sudden bistable transition between epithelial and mesenchymal states. The mutant, however, shows a much more gradual transition. This also fits some of the experimental results of [38]. This result also shows that self-sustaining feedback loops (stable motifs) don't necessarily lose their nonlinear attributes with some added noise. 


\section{Reduced CyclinB-Cdk1 induced apoptosis improves asynchronous cell cycle model}

In 2019 we published an 89 node modular Boolean cell cycle model, which explained a wide range of healthy and pathological cell cycle behaviors, from the aberrant cell cycle driven by hyperactive PI3K, to the different effects of timed knockout of Polo-kinase 1 (Plk1), such as mitotic catastrophe or polyploidy [29]. The model has the most robust cyclic behavior when simulated with the synchronous update scheme. In Sizek et al. [29] we also considered multiple kinds of asynchronous update schemes and found that the model had a few non-biological behaviors when simulated with the general asynchronous update scheme. These behaviors were resolved when using a biased order asynchronous update scheme, which guaranteed that a critical subset of node updates are made in their biologically observed order. However, the biased order asynchronous update scheme needs a number of additional parameters and its implementation is rather non-intuitive and hard to link to biology in a straightforward way.

Here we propose a simpler solution to some of the model's problems posed by the general asynchronous update scheme and a few PEW operators, while also keeping the wide range of model behaviors that match experimental data.

In Figure 4 we show an extended ensemble simulation of the original cell cycle model with constant high growth factor stimulation. This model focuses on cell cycle dynamics and assumes healthy growth conditions, i.e. no external damage or perturbation is induced. The cell cycle driver kinases and cyclins turn on early and drive the cell cycle, however, one can notice that the apoptotic nodes, such as Casp3, Casp2, and most notably CAD (which signals the execution of apoptosis) gradually increase in time - i.e. more and more cells in the ensemble die. This is due to the several faulty behaviors related to the update scheme, which ultimately lead to apoptosis instead of continued cycling. These behaviors are discussed in Sizek et al. [29] in more detail, along with ways to overcome them using a biased update scheme.

Most often the unexpected apoptosis is the result of the perturbed balance between pro- and anti-apoptotic influences during metaphase. In metaphase cells are sensitized to apoptosis in the event of a failure to assemble the mitotic spindle and pass the spindle assembly checkpoint. There is evidence that the CyclinB-Cdk1 complex phosphorylates a part of the pool of anti-apoptotic proteins, such as MCL-1, BCLXL, and BCL2. A prolonged mitotic phase leads to a high degree of phosphorylation, which then degrades the anti-apoptotic proteins and initiates apoptosis [40-43]. This happens very rarely in healthy cells, yet with asynchronous updating, the in-silico time scales at which the event occurs are often not proportional (significantly shorter) to the time scales in real cells. To counter this we propose a weakening of the inhibitory links from the CyclinB-Cdk1 complex to the anti-apoptotic links. This modification will have no effect on the incredibly important role of the CyclinB-Cdk1 complex in driving the cell cycle, nor 
will it completely remove its influence on the anti-apoptotic nodes. Also, we did not alter the protective (anti-apoptotic) effects Cdk1/CyclinB has during normal cell cycle progression, where it blocks Caspase 2 activity. This means that if the cycle is indeed stopped during mitosis due to external damage, the CyclinB-Cdk1 complex can still prime cells for apoptosis, only it will take relatively more time (update steps). This, however, should happen very rarely in wild-type behavior (no external perturbation).

In Figure 4 (bottom) we show the effect of introducing a [p_on $=0.5, p \_$off $=0$ ] weight on three hyperedges from the CyclinB and Cdk1 complex to the three anti-apoptotic nodes mentioned above (MCL-1, BCLXL, and BCL2). Due to this modification, the number of cells committing to apoptosis during the same time period drops to half. In Figure 5 we present a more detailed analysis of how the ratio of apoptotic cells changes as a function of the p_on parameter. One can notice that a 50\% reduction in the CAD activity can be achieved with p_on $=0.6$ and further weakening the links (which might compromise their intended function) no longer reduces the CAD activity in a significant way.

It is beyond the scope of this paper to address all the issues that are caused by the noisy update scheme in this cell cycle model. The fact that a nonzero fraction of cells still commit to apoptosis without simulated damage is due to other unintended effects of the general asynchronous update, such as aneuploidy and skipped cytokinesis. (For further details on the model's errors and explanations see Suppl. Figure 6 of [29]).

However, one can see from these simple examples, that with slight modifications of edge weights one can achieve a high degree of improvement in the quality of the emergent behavior. The method achieves this by effectively reducing the number of non-biological update orders otherwise allowed by the general asynchronous update scheme, which is especially problematic when in the real system different links work on significantly different time scales. 

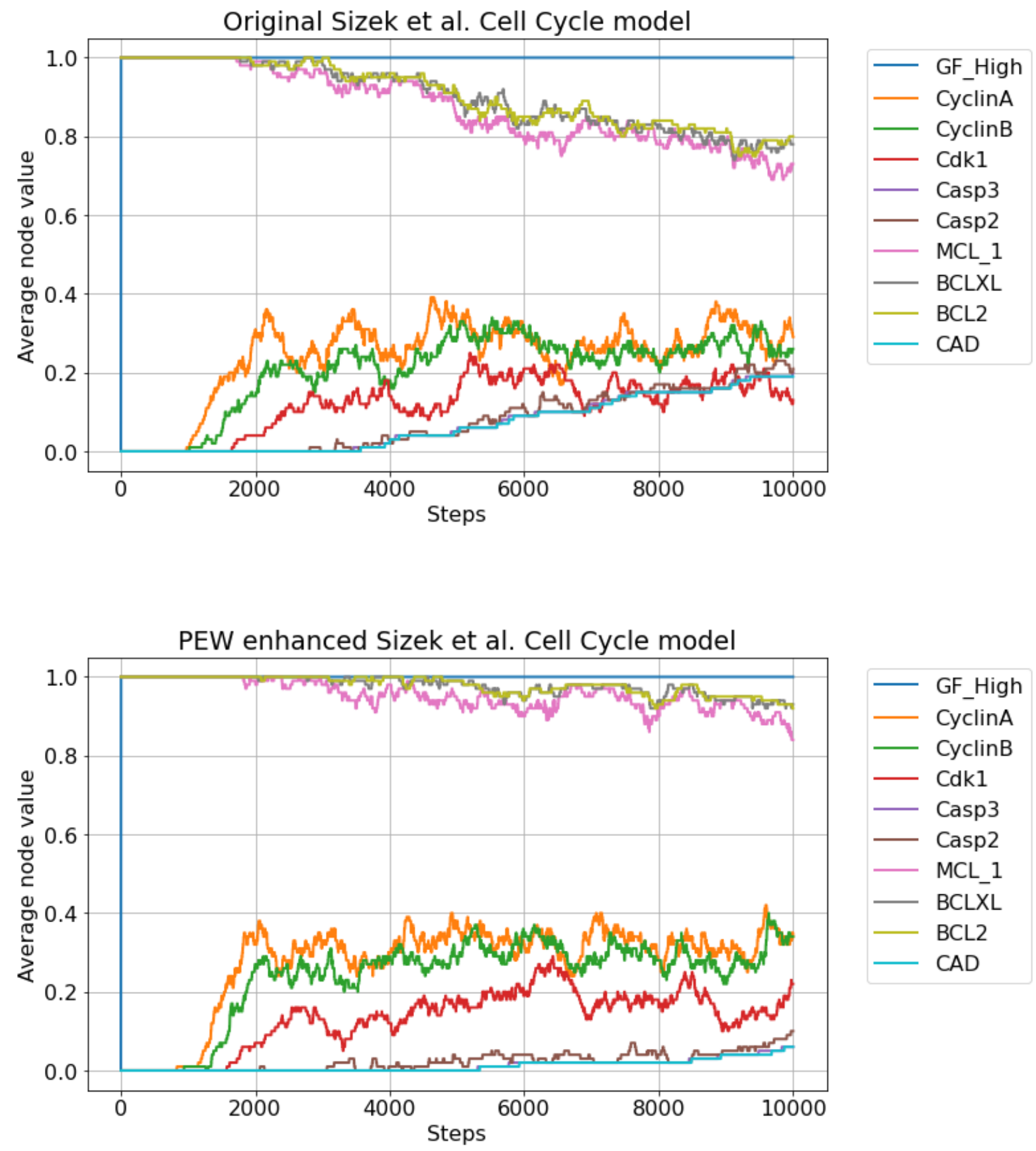

Figure 4: The rate of cells committing to unsolicited apoptosis drops significantly after the introduction of PEW operators. The figures show the average node values of 100 independently simulated Cell Cycle models with High growth factor stimulation and no external damage. On the top figure (original model) after 10000 steps, roughly $20 \%$ of the cells have 
CAD active, meaning that they committed to apoptosis. On the bottom figure (PEW operators added), this rate is about half of that. The same difference in apoptosis commitment rate is also visible in the evolution of anti-apoptotic nodes (MCL-1, BCLXL, BCL2). Otherwise, there is no qualitative difference in the cell cycle progression (the cell cycle markers such as CyclinA and CyclinB still fluctuate within the same boundaries, i.e. the same proportion of cells are active in the cell cycle over time).

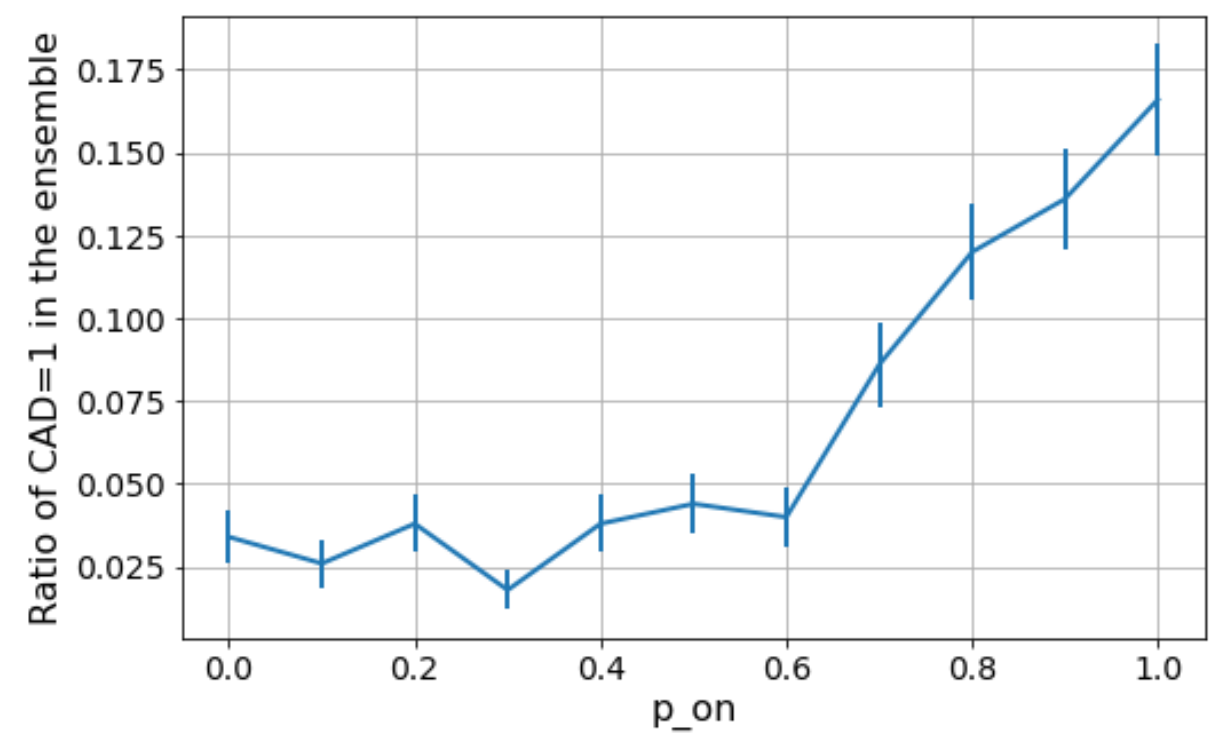

Figure 5. The propensity of $C A D=1$ (apoptosis commitment) drops with the decrease of the p_on (increase in state-dependent noise) on the selected edges. Each data point represents a simulation done on an ensemble of 500 models for each p_on value with 10000 simulation steps, as a function of $p_{-}$on in the PEW operator [p_on, 0] acting on the CyclinB+Cdk1 complex's links inhibiting the anti-apoptotic nodes (MCL-1, BCLXL, and BCL2). The same p_on value is applied for all three hyperedges (CyclinB and Cdk1 clauses). The "default" behavior is at p_on=1, while $p_{-}$on $=0$ is the special case of severing the link with the clause "Cdk1 and CyclinB" always being 0. Error bars represent the standard error within the ensemble.

\section{Discussion}

In this paper, we have presented a method of introducing different levels of state-dependent edge-noise into Boolean models of biological systems via probabilistic weight operators. The goal of this method is not necessarily restricted to modeling the intrinsic stochasticity of systems (even though adding general noise is possible within this framework). The canonical stochastic methods discussed in the paper have a wide variety of ways of introducing system-wide as well as node-level biological noise. The goal of the PEW method is threefold.

First, to introduce stochasticity to the level of edges and thus introduce relative weight to edges and hyperedges through noise operators. With this, one can emulate very specific mutations, 
changes in binding site affinities, differences in interaction time scales, etc., and also offer a way to implement edge-level uncertainty.

Second, to offer a technical implementation for in-silico simulations which is easy to use; thus the method is implemented as an extended version of the well-established BooleanNet tool [32]. The third goal is to reproduce the noisy dynamics of several canonical methods (discussed in more detail in Appendix 1). Thus, the PEW framework shouldn't be thought of as a new modeling framework, instead, as a toolkit for fine-tuning Boolean models with the targeted placement of PEW operators. Using our method one can introduce an increased uncertainty for certain connections, or conversely given a non-zero base noise, increase the relative certainty of regulations and even emulate a short-term adaptation akin to cellular learning. Cellular learning has been increasingly discussed in recent studies as a way of cells acclimatizing to repeated signals with faster phenotype convergence [44]. In this framework, we can think about learning as the reduction of noise, i.e. one could model the more optimal reaction of learning cell cultures with changing weights from noisier to less noisy parameters. Changing levels of noise within positive feedback loops could be especially effective, as we have shown in the application to the EMT study, where a less noisy edge (Zeb - miR200 in the wild type case) produced a faster epithelial convergence than the more noisy edge (mutant). In fact, there is evidence to suggest that intrinsically disordered proteins (IDPs) do exactly that, namely, acclimatize to repeated signals and shape their originally disordered (more noisy) structure to one more responsive to the signal (less noisy) [44-47].

Another reason to target self-sustaining positive feedback loops (stable motifs) with PEW operators is to counteract their intrinsic irreversibility in permanently committing the system to certain paths. For example, autocrine signaling loops involving the secretion of signaling molecules that drive their own production can create stable motifs that irreversibly lock a modeled cell into a particular self-sustaining state. Inherent in such a model is the assumption that a cell secretes a sufficiently strong signal to saturate its own autocrine signaling pathway; an assumption that may not be realistic in micro-environments that do not help concentrate these signals [22]. With PEW operators one can implement this effect without losing the nonlinear effects of feedback mechanisms, as we have shown in the EMT commitment. Even having a single link made slightly noisier in a stable motif can act as a natural decay parameter as it gradually unlocks a self-sustaining feedback loop and allows different pathways to engage.

One more potential application is encoding uncertainty in data-driven regulatory network building. Many of the emerging network medicine methods generate weighted networks from combining information from different omics data sources $[48,49]$. Combining expression data with such regulatory networks in order to produce dynamic models is an important challenge as more and more clinical research is focused on finding therapeutic targets through the control of dynamical networks. Binarizing the weights of such data-driven networks might involve a lot of costly compromises, thus having a way of encoding edge-weights in the form of noise in a dynamic model could be a handy tool.

We would like to emphasize that this method is not necessarily restricted to the Boolean case. Indeed a case using continuous variables was already implemented to reproduce the results of 
[28] (see Supplementary Jupyter notebook). Moreover, discrete, multi-level versions are also possible. In this case, however, one might want a different weight parameter for all possible node values if the state-dependent aspect of the method is meant to be kept.

Finally, this paper does not offer any concrete way to methodically determine the weights in the PEW operators. We believe that this is a highly context-dependent task and if the number of PEW operators introduced in a model is relatively low (like in the applications presented in this paper) the weights can be fine-tuned manually. Having too many PEW operators without a systemic source for the weights eventually will pose the same problems ODE models face, of having to fine-tune too many parameters. The collective behavior of many interacting PEW-enhanced edges should be the subject of further research.

In this paper, we introduced probabilistic edge-weights into Boolean dynamic network models and showed that they successfully model system noise making the dynamic predictions more accurate. Moreover, we developed the PEW framework, a simple-to-use implementation of this methodology in the widely used BooleanNet program package. Probabilistic edge-weight operators may open new routes to understanding the cellular learning process, as well as to include omics data to Boolean network dynamics models.

\section{Author Contributions}

DD, NK and PCs conceived the study, DD developed the methodology, implemented the computations, developed the applications. DD, NK, PCs wrote the paper.

\section{Acknowledgments}

The authors thank Prof. Réka Albert and Prof. Erzsébet Ravasz Regan for their feedback and insightful comments that significantly improved the paper.

This work was supported by the Hungarian National Research, Development and Innovation Office (K131458), by the Higher Education Institutional Excellence Programme of the Ministry of Human Capacities in Hungary, within the framework of the Molecular Biology thematic programs of Semmelweis University, by the Thematic Excellence Programme (Tématerületi Kiválósági Program, 2020-4.1.1.-TKP2020, TKP2021-EGA-24) of the Ministry for Innovation and Technology in Hungary, within the framework of the Molecular Biology thematic program of the Semmelweis University.

\section{Appendix 1}

Other Noisy Boolean models as special cases of PEW 


\section{BNp with perturbation probability $p$}

Reproducing perturbed Boolean Networks within the PEW framework is straightforward, by treating the full Boolean rule of a node as a hyper-edge and assigning $p$ and $1-p$ Bernoulli noise for the OFF and ON states respectively. For a single node, this means applying the PEW operator on the full rule such as:

$$
\begin{aligned}
& \sigma_{v_{i}}(t+1)=f_{i}\left(\boldsymbol{\sigma}_{\operatorname{Reg}\left(v_{i}\right)}(t)\right), \text { becomes: } \\
& \sigma_{v_{i}}(t+1)=P\left(1-p, p, f_{\text {Bernoulli }}\right) f_{i}\left(\boldsymbol{\sigma}_{\operatorname{Reg}\left(v_{i}\right)}(t)\right)
\end{aligned}
$$

The PEW framework offers the added flexibility that one does not have to use the same $p$ probability of every node, in fact, all nodes can have a different noise distribution.

SDDS with probabilities $p_{u p}, p_{\text {down }}$

$$
\begin{aligned}
& \sigma_{v_{i}}(t+1)=f_{i}\left(\boldsymbol{\sigma}_{\operatorname{Reg}\left(v_{i}\right)}(t)\right) \\
& \sigma_{v_{i}}(t+1)=P\left(p_{\text {up }}, 1-p_{\text {down }}, f_{\text {Bernoulli }}\left(\sigma_{v_{i}}(t)\right)\right) f_{i}\left(\boldsymbol{\sigma}_{\operatorname{Reg}\left(v_{i}\right)}(t)\right)
\end{aligned}
$$

In this case $f$ also depends on the present value of the node $i$ and the conditional parameter is decided based on the comparison between $\sigma_{v_{i}}(t)$ and $f_{i}\left(\boldsymbol{\sigma}_{\operatorname{Reg}\left(v_{i}\right)}(t)\right)$

For the reproduction of one of the results from Murrugarra et al. [19] see the Suppl Jupyter notebook.

\section{Difference between PBNs and PEWBNs}

As opposed to PBNs, the PEW enhanced BNs still have a single unique function per node, however, thanks to the PEW operator the outcome is indeed probabilistic. This, in some cases, can be expressed in the form of a PBN, where every edge-weight combination is expressed as a separate function with the weights distributed as probabilities for picking the different functions. However, a key difference between PBNs and PEWBNs is that while in the case of PBNs the functions are chosen based on a pre-determined probability distribution, in the case of PEWBNs the function parameters are conditional on the value of the source-node of the edge.

Using more complex $f$ weight functions than the Bernoulli coin-toss the relationship between PBNs and PewBNs becomes even more complex.

\section{PEWBNs are a special case of DBNs}

We would argue that the PEW method is a step toward the DBN framework in generality because the rules of some nodes with PEWs become stochastic and conditionally dependent on the value of the (subset of) parents. Nonetheless, it's still more pragmatic to view PEW models 
as a separate framework because, as we show in the empirical applications, probabilistic edge weights are meant as very specific, targeted modifications to Boolean models and are easily made compatible with existing tools of Boolean network analysis. On the other hand, DBNs are likely the most general modeling framework of which PEWs models represent a special case.

\section{Appendix 2}

\section{Boolean rules with and without PEW operators}

\section{Application 1 - the simplified EMT model:}

SNAI1 positive feedback loop - SNAI1 inhibits transcription of miR-34a [50], which in turn inhibits SNAI1 translation resulting in decreased SNAI1 mRNA and protein levels [51,52]. Additionally, SNAI1 also inhibits HNF4A transcription [53], which positively regulates miR-122 transcription [54], and miR-122, in turn, inhibits SNAl1 translation [55].

TGFB - miR200 inhibition - Non-canonical TGFB pathway results in PI3K/AKT pathway activation [56], leading to decreased GSK3B nuclear localization [56,57] nuclear GSK3B-mediated DNMT1 phosphorylation and degradation [58,59], thus decreasing miR-200 promoter methylation [60].

The remaining interactions in the simplified model are all canonical and supported by multiple papers referenced in the main text, such as [6,37-39].

\section{Wild type model:}

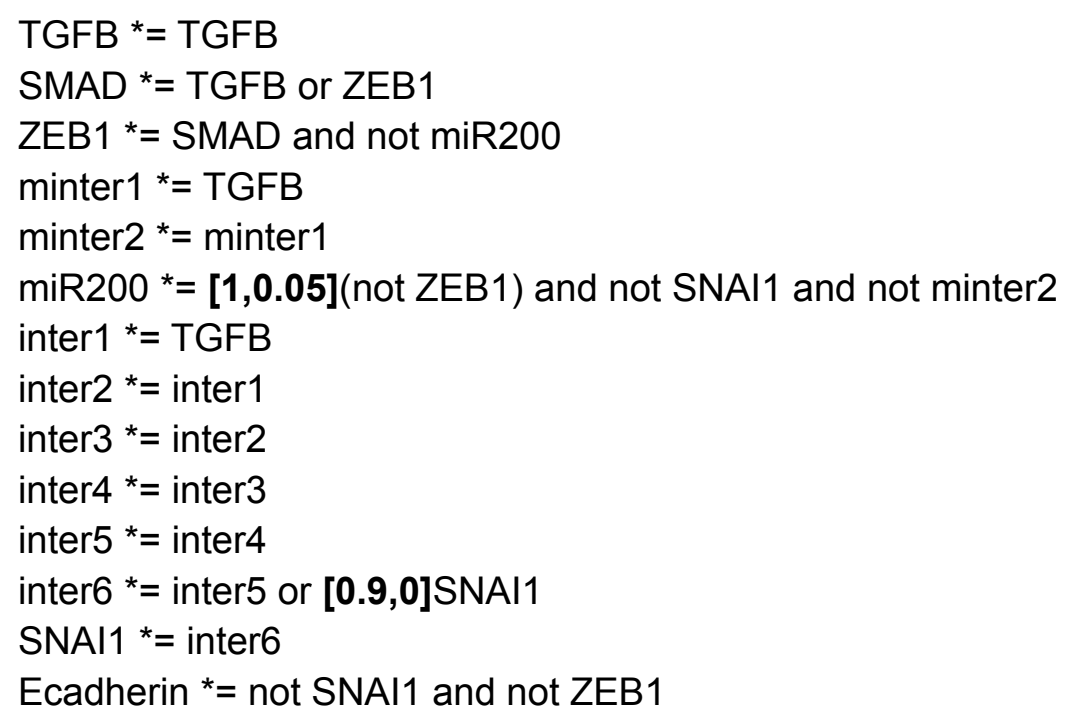


Mutant model (the only difference to compared to the wild type is in the PEW parameters):

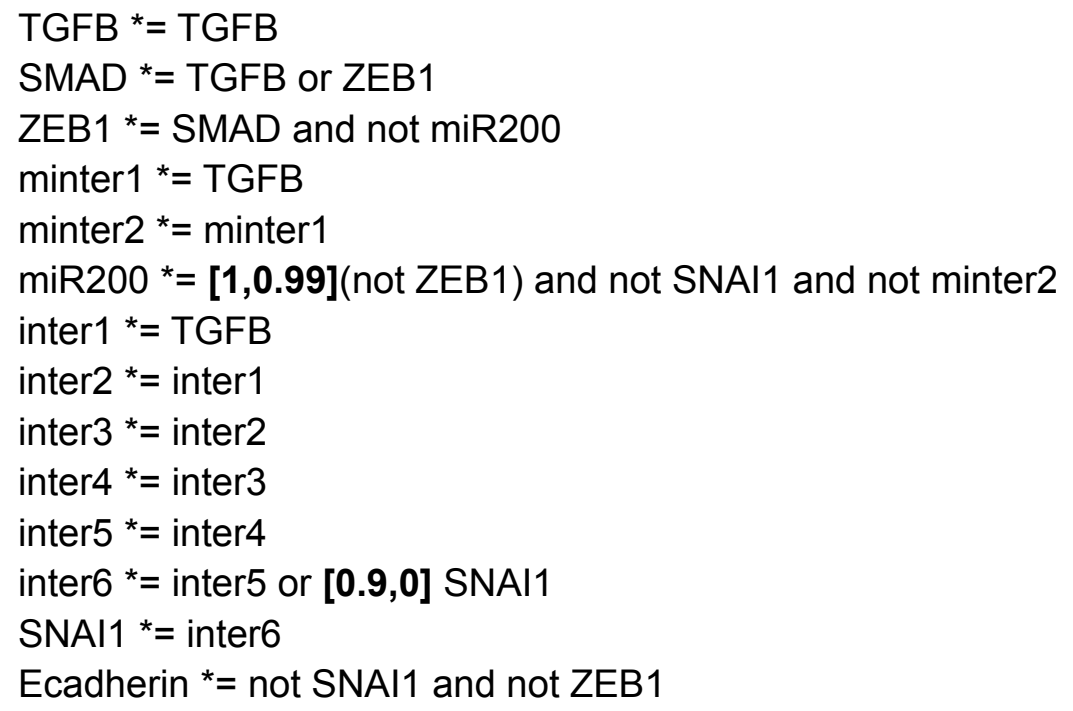

Application 2 - Sizek et al. model (for all Boolean rules and citations see $\underline{\text { SI Table } 1}$ of [29])

MCL_1 ${ }^{*}=$ not Casp3 and not Casp2 and (not GSK3 or (AKT_B and (ERK or not E2F1)) ) and (not ((Cdk1 and CyclinB) and U_Kinetochores) )

$\mathrm{BCLXL}{ }^{*}=$ not Casp3 and (BCL2 or not BAD) and ( not U_Kinetochores or ( PIk1 and (not (CyclinB and Cdk1) or (BCL2 and MCL_1) ) ) or ( (BCL2 and MCL_1) and not (CyclinB and Cdk1) ) )

$\mathrm{BCL} 2{ }^{*}=$ not $($ Casp3 or BAD or BIM or BIK) and ( not U_Kinetochores or (MCL_1 and BCLXL) or (PIk1 and (BCLXL or MIAPsCL_1 or not (Cdk1 and CyclinB) ) ) )

\section{With PEW operators:}

MCL_1 ${ }^{*}=$ not Casp3 and not Casp2 and (not GSK3 or (AKT_B and (ERK or not E2F1)) ) and (not $([0.5,0](C d k 1$ and CyclinB) and U_Kinetochores) )

$\mathrm{BCLXL}{ }^{*}=$ not Casp3 and (BCL2 or not BAD) and ( not U_Kinetochores or ( Plk1 and (not $[0.5,0]$ (CyclinB and Cdk1) or (BCL2 and MCL_1) ) ) or ( (BCL2 and MCL_1) and not (CyclinB and Cdk1) ) )

$\mathrm{BCL} 2{ }^{*}=$ not $($ Casp3 or BAD or BIM or BIK) and ( not U_Kinetochores or (MCL_1 and BCLXL) or (PIk1 and (BCLXL or MIAPsCL_1 or not [0.5,0] (Cdk1 and CyclinB) ) ) )

\section{Code availability}


The modified version BooleanNet [10] along with the Jupyter notebooks containing the code for all the results presented in this paper (Applications.ipynb), as well as the ways to reproduce some of the other noisy models (Murrugarra et al 2012 paper results.ipynb and Poret et al paper results-Boolean init.ipynb) are available on the following GitHub page: https://github.com/deriteidavid/boolean2pew.

\section{References}

1. Albert R, Othmer HG. The topology of the regulatory interactions predicts the expression pattern of the segment polarity genes in Drosophila melanogaster. J Theor Biol. 2003;223: $1-18$.

2. Wang R-S, Saadatpour A, Albert R. Boolean modeling in systems biology: an overview of methodology and applications. Physical Biology. 2012. p. 055001. doi:10.1088/1478-3975/9/5/055001

3. Faure A, Naldi A, Chaouiya C, Thieffry D. Dynamical analysis of a generic Boolean model for the control of the mammalian cell cycle. Bioinformatics. 2006. pp. e124-e131. doi:10.1093/bioinformatics/btl210

4. Regan ER, Aird WC. Dynamical systems approach to endothelial heterogeneity. Circ Res. 2012;111: 110-130.

5. Zhang R, Shah MV, Yang J, Nyland SB, Liu X, Yun JK, et al. Network model of survival signaling in large granular lymphocyte leukemia. Proc Natl Acad Sci U S A. 2008;105: 16308-16313.

6. Steinway SN, Zañudo JGT, Ding W, Rountree CB, Feith DJ, Loughran TP Jr, et al. Network modeling of TGF $\beta$ signaling in hepatocellular carcinoma epithelial-to-mesenchymal transition reveals joint sonic hedgehog and Wnt pathway activation. Cancer Res. 2014;74: 5963-5977.

7. Deritei D, Aird WC, Ercsey-Ravasz M, Regan ER. Principles of dynamical modularity in biological regulatory networks. Sci Rep. 2016;6: 21957.

8. Shreim A, Berdahl A, Greil F, Davidsen J, Paczuski M. Attractor and basin entropies of random Boolean networks under asynchronous stochastic update. Phys Rev E Stat Nonlin Soft Matter Phys. 2010;82: 035102.

9. Saadatpour A, Albert I, Albert R. Attractor analysis of asynchronous Boolean models of signal transduction networks. J Theor Biol. 2010;266: 641-656.

10. Saadatpour A, Wang R-S, Liao A, Liu X, Loughran TP, Albert I, et al. Dynamical and Structural Analysis of a $\mathrm{T}$ Cell Survival Network Identifies Novel Candidate Therapeutic Targets for Large Granular Lymphocyte Leukemia. PLoS Computational Biology. 2011. p. e1002267. doi:10.1371/journal.pcbi.1002267

11. Fumiã HF, Martins ML. Boolean network model for cancer pathways: predicting carcinogenesis and targeted therapy outcomes. PLoS One. 2013;8: e69008. 
12. Zañudo JGT, Albert R. An effective network reduction approach to find the dynamical repertoire of discrete dynamic networks. Chaos. 2013;23: 025111.

13. Deritei D, Rozum J, Ravasz Regan E, Albert R. A feedback loop of conditionally stable circuits drives the cell cycle from checkpoint to checkpoint. Sci Rep. 2019;9: 16430.

14. Zañudo JGT, Yang G, Albert R. Structure-based control of complex networks with nonlinear dynamics. Proc Natl Acad Sci U S A. 2017;114: 7234-7239.

15. Zañudo JGT, Albert R. Cell fate reprogramming by control of intracellular network dynamics. PLoS Comput Biol. 2015;11: e1004193.

16. Steinway SN, Zañudo JGT, Michel PJ, Feith DJ, Loughran TP, Albert R. Combinatorial interventions inhibit TGF $\beta$-driven epithelial-to-mesenchymal transition and support hybrid cellular phenotypes. NPJ Syst Biol Appl. 2015;1: 15014.

17. Shmulevich I, Dougherty ER, Kim S, Zhang W. Probabilistic Boolean Networks: a rule-based uncertainty model for gene regulatory networks. Bioinformatics. 2002;18: 261-274.

18. Murphy K, Mian S. Modelling gene expression data using dynamic Bayesian networks. Technical report, Computer Science Division, University of California, Berkeley, CA. 1999;104.

19. Murrugarra D, Veliz-Cuba A, Aguilar B, Arat S, Laubenbacher R. Modeling stochasticity and variability in gene regulatory networks. EURASIP J Bioinform Syst Biol. 2012;2012: 5.

20. Shmulevich I, Dougherty ER. Probabilistic Boolean Networks: The Modeling and Control of Gene Regulatory Networks. SIAM; 2010.

21. Elowitz MB. Stochastic Gene Expression in a Single Cell. Science. 2002. pp. 1183-1186. doi:10.1126/science.1070919

22. Blake WJ, Balázsi G, Kohanski MA, Isaacs FJ, Murphy KF, Kuang Y, et al. Phenotypic consequences of promoter-mediated transcriptional noise. Mol Cell. 2006;24: 853-865.

23. Urchueguía A, Galbusera L, Chauvin D, Bellement G, Julou T, van Nimwegen E. Genome-wide gene expression noise in Escherichia coli is condition-dependent and determined by propagation of noise through the regulatory network. PLoS Biol. 2021;19: e3001491.

24. Bornholdt S. Boolean network models of cellular regulation: prospects and limitations. J R Soc Interface. 2008;5 Suppl 1: S85-94.

25. Ladbury JE, Arold ST. Noise in cellular signaling pathways: causes and effects. Trends Biochem Sci. 2012;37: 173-178.

26. Balázsi G, van Oudenaarden A, Collins JJ. Cellular decision making and biological noise: from microbes to mammals. Cell. 2011;144: 910-925.

27. Trairatphisan P, Mizera A, Pang J, Tantar AA, Schneider J, Sauter T. Recent development and biomedical applications of probabilistic Boolean networks. Cell Commun Signal. 2013;11: 46. 
28. Poret A, Sousa CM, Boissel J-P. Enhancing Boolean networks with fuzzy operators and edge tuning. 2014. Available: https://hal.archives-ouvertes.fr/hal-01018236/

29. Sizek H, Hamel A, Deritei D, Campbell S, Ravasz Regan E. Boolean model of growth signaling, cell cycle and apoptosis predicts the molecular mechanism of aberrant cell cycle progression driven by hyperactive PI3K. PLoS Comput Biol. 2019;15: e1006402.

30. Guberman E, Sherief H, Regan ER. Boolean model of anchorage dependence and contact inhibition points to coordinated inhibition but semi-independent induction of proliferation and migration. Comput Struct Biotechnol J. 2020;18: 2145-2165.

31. Berge C. Hypergraphs: Combinatorics of Finite Sets. Elsevier; 1984.

32. Albert I, Thakar J, Li S, Zhang R, Albert R. Boolean network simulations for life scientists. Source Code Biol Med. 2008;3: 16.

33. Johnson SC, Others. Yacc: Yet another compiler-compiler. Bell Laboratories Murray Hill, NJ; 1975.

34. Kalluri R, Weinberg RA. The basics of epithelial-mesenchymal transition. J Clin Invest. 2009;119: 1420-1428.

35. Kim D, Xing T, Yang Z, Dudek R, Lu Q, Chen Y-H. Epithelial Mesenchymal Transition in Embryonic Development, Tissue Repair and Cancer: A Comprehensive Overview. Journal of Clinical Medicine. 2017. p. 1. doi:10.3390/jcm7010001

36. Nieto MA. The ins and outs of the epithelial to mesenchymal transition in health and disease. Annu Rev Cell Dev Biol. 2011;27: 347-376.

37. Gregory PA, Bert AG, Paterson EL, Barry SC, Tsykin A, Farshid G, et al. The miR-200 family and miR-205 regulate epithelial to mesenchymal transition by targeting ZEB1 and SIP1. Nat Cell Biol. 2008;10: 593-601.

38. Celià-Terrassa T, Bastian C, Liu DD, Ell B, Aiello NM, Wei Y, et al. Hysteresis control of epithelial-mesenchymal transition dynamics conveys a distinct program with enhanced metastatic ability. Nature Communications. 2018. 9,5005 doi:10.1038/s41467-018-07538-7

39. Gregory PA, Bracken CP, Smith E, Bert AG, Wright JA, Roslan S, et al. An autocrine TGF-beta/ZEB/miR-200 signaling network regulates establishment and maintenance of epithelial-mesenchymal transition. Mol Biol Cell. 2011;22: 1686-1698.

40. Harley ME, Allan LA, Sanderson HS, Clarke PR. Phosphorylation of Mcl-1 by CDK1-cyclin B1 initiates its Cdc20-dependent destruction during mitotic arrest. EMBO J. 2010;29: 2407-2420.

41. Terrano DT, Upreti M, Chambers TC. Cyclin-dependent kinase 1-mediated Bcl-xL/Bcl-2 phosphorylation acts as a functional link coupling mitotic arrest and apoptosis. Mol Cell Biol. 2010;30: 640-656.

42. Zhou L, Cai X, Han X, Xu N, Chang DC. CDK1 switches mitotic arrest to apoptosis by phosphorylating $\mathrm{Bcl}-2 / \mathrm{Bax}$ family proteins during treatment with microtubule interfering agents. Cell Biol Int. 2014;38: 737-746. 
43. Eichhorn JM, Sakurikar N, Alford SE, Chu R, Chambers TC. Critical role of anti-apoptotic $\mathrm{Bcl}-2$ protein phosphorylation in mitotic death. Cell Death Dis. 2013;4: e834.

44. Csermely P, Kunsic N, Mendik P, Kerestély M, Faragó T, Veres DV, et al. Learning of Signaling Networks: Molecular Mechanisms. Trends Biochem Sci. 2020;45: 284-294.

45. Ferguson KM, Berger MB, Mendrola JM, Cho HS, Leahy DJ, Lemmon MA. EGF activates its receptor by removing interactions that autoinhibit ectodomain dimerization. Mol Cell. 2003;11: 507-517.

46. Csermely P, Palotai R, Nussinov R. Induced fit, conformational selection and independent dynamic segments: an extended view of binding events. Trends Biochem Sci. 2010;35: 539-546.

47. Mooney SM, Jolly MK, Levine $\mathrm{H}$, Kulkarni P. Phenotypic plasticity in prostate cancer: role of intrinsically disordered proteins. Asian J Androl. 2016;18: 704-710.

48. Glass K, Huttenhower C, Quackenbush J, Yuan G-C. Passing messages between biological networks to refine predicted interactions. PLoS One. 2013;8: e64832.

49. Kuijjer ML, Glass K. Reconstructing Sample-Specific Networks using LIONESS. doi:10.1101/2021.09.27.461954

50. Siemens H, Jackstadt R, Hünten S, Kaller M, Menssen A, Götz U, et al. miR-34 and SNAIL form a double-negative feedback loop to regulate epithelial-mesenchymal transitions. Cell Cycle. 2011;10: 4256-4271.

51. Kim NH, Kim HS, Li X-Y, Lee I, Choi H-S, Kang SE, et al. A p53/miRNA-34 axis regulates Snail1-dependent cancer cell epithelial-mesenchymal transition. J Cell Biol. 2011;195: 417-433.

52. Yan X, Zhang D, Wu W, Wu S, Qian J, Hao Y, et al. Mesenchymal Stem Cells Promote Hepatocarcinogenesis via IncRNA-MUF Interaction with ANXA2 and miR-34a. Cancer Res. 2017;77: 6704-6716.

53. Yang M, Li S-N, Anjum KM, Gui L-X, Zhu S-S, Liu J, et al. A double-negative feedback loop between Wnt- $\beta$-catenin signaling and HNF4 $\alpha$ regulates epithelial-mesenchymal transition in hepatocellular carcinoma. J Cell Sci. 2013;126: 5692-5703.

54. Li Z-Y, Xi Y, Zhu W-N, Zeng C, Zhang Z-Q, Guo Z-C, et al. Positive regulation of hepatic miR-122 expression by HNF4a. J Hepatol. 2011;55: 602-611.

55. Jin Y, Wang J, Han J, Luo D, Sun Z. MiR-122 inhibits epithelial-mesenchymal transition in hepatocellular carcinoma by targeting Snail1 and Snail2 and suppressing WNT/ $\beta$-cadherin signaling pathway. Exp Cell Res. 2017;360: 210-217.

56. Hamidi A, Song J, Thakur N, Itoh S, Marcusson A, Bergh A, et al. TGF- $\beta$ promotes PI3K-AKT signaling and prostate cancer cell migration through the TRAF6-mediated ubiquitylation of p85a. Sci Signal. 2017;10. doi:10.1126/scisignal.aal4186

57. Bautista SJ, Boras I, Vissa A, Mecica N, Yip CM, Kim PK, et al. mTOR complex 1 controls the nuclear localization and function of glycogen synthase kinase $3 \beta$. J Biol Chem. 2018;293: 14723-14739. 
58. Lin R-K, Hsieh Y-S, Lin P, Hsu H-S, Chen C-Y, Tang Y-A, et al. The tobacco-specific carcinogen NNK induces DNA methyltransferase 1 accumulation and tumor suppressor gene hypermethylation in mice and lung cancer patients. J Clin Invest. 2010;120: 521-532.

59. Fang Q-L, Yin Y-R, Xie C-R, Zhang S, Zhao W-X, Pan C, et al. Mechanistic and biological significance of DNA methyltransferase 1 upregulated by growth factors in human hepatocellular carcinoma. Int J Oncol. 2015;46: 782-790.

60. Sui C-J, Zhou Y-M, Shen W-F, Dai B-H, Lu J-J, Zhang M-F, et al. Long noncoding RNA GIHCG promotes hepatocellular carcinoma progression through epigenetically regulating miR-200b/a/429. J Mol Med . 2016;94: 1281-1296. 